\title{
Existence of Periodic Solutions for a Delayed Ratio-Dependent Three-Species Predator-Prey Diffusion System on Time Scales
}

\author{
Zhenjie Liu \\ School of Mathematics and Computer, Harbin University, Harbin, Heilongjiang 150086, China \\ Correspondence should be addressed to Zhenjie Liu, liouj2008@126.com
}

Received 3 September 2008; Accepted 21 January 2009

Recommended by Binggen Zhang

This paper investigates the existence of periodic solutions of a ratio-dependent predator-prey diffusion system with Michaelis-Menten functional responses and time delays in a two-patch environment on time scales. By using a continuation theorem based on coincidence degree theory, we obtain suffcient criteria for the existence of periodic solutions for the system. Moreover, when the time scale $\mathbb{T}$ is chosen as $\mathbb{R}$ or $\mathbb{Z}$, the existence of the periodic solutions of the corresponding continuous and discrete models follows. Therefore, the methods are unified to provide the existence of the desired solutions for the continuous differential equations and discrete difference equations.

Copyright (C) 2009 Zhenjie Liu. This is an open access article distributed under the Creative Commons Attribution License, which permits unrestricted use, distribution, and reproduction in any medium, provided the original work is properly cited.

\section{Introduction}

The traditional predator-prey model has received great attention from both theoretical and mathematical biologists and has been studied extensively (e.g., see [1-4] and references therein). Based on growing biological and physiological evidences, some biologists have argued that in many situations, especially when predators have to search for food (and therefore, have to share or compete for food), the functional response in a prey-predator model should be ratio-dependent, which can be roughly stated as that the per capita predator growth rate should be a function of the ratio of prey to predator abundance. Starting from this argument and the traditional prey-dependent-only mode, Arditi and Ginzburg [5] first proposed the following ratio-dependent predator-prey model:

$$
\begin{aligned}
& \dot{x}=x(a-b x)-\frac{c x y}{m y+x} \\
& \dot{y}=y\left(-d+\frac{f x}{m y+x}\right)
\end{aligned}
$$


which incorporates mutual interference by predators, where $g(x)=c x /(m y+x)$ is a Michaelis-Menten type functional response function. Equation (1.1) has been studied by many authors and seen great progress (e.g., see [6-11]).

$\mathrm{Xu}$ and Chen [11] studied a delayed two-predator-one-prey model in two patches which is described by the following differential equations:

$$
\begin{aligned}
x_{1}^{\prime}(t)= & x_{1}(t)\left(a_{1}-a_{11} x_{1}(t)-\frac{a_{13} x_{3}(t)}{m_{13} x_{3}(t)+x_{1}(t)}-\frac{a_{14} x_{4}(t)}{m_{14} x_{4}(t)+x_{1}(t)}\right) \\
& +D_{1}(t)\left(x_{2}(t)-x_{1}(t)\right), \\
x_{2}^{\prime}(t)= & x_{2}(t)\left(a_{2}-a_{22} x_{2}(t)\right)+D_{2}(t)\left(x_{1}(t)-x_{2}(t)\right), \\
x_{3}^{\prime}(t)= & x_{3}(t)\left(-a_{3}+\frac{a_{31} x_{1}\left(t-\tau_{1}\right)}{m_{13} x_{3}\left(t-\tau_{1}\right)+x_{1}\left(t-\tau_{1}\right)}\right), \\
x_{4}^{\prime}(t)= & x_{4}(t)\left(-a_{4}+\frac{a_{41} x_{1}\left(t-\tau_{2}\right)}{m_{14} x_{4}\left(t-\tau_{2}\right)+x_{1}\left(t-\tau_{2}\right)}\right) .
\end{aligned}
$$

In view of periodicity of the actual environment, Huo and Li [12] investigated a more general delayed ratio-dependent predator-prey model with periodic coefficients of the form

$$
\begin{aligned}
& \dot{x}_{1}(t)=x_{1}(t)\left(a_{1}(t)-a_{11}(t) x_{1}\left(t-\tau_{11}\right)-\frac{a_{12}(t) x_{2}(t)}{m_{1}+x_{1}(t)}\right) \\
& \dot{x}_{2}(t)=x_{2}(t)\left(-a_{2}(t)+\frac{a_{21}(t) x_{1}\left(t-\tau_{21}\right)}{m_{1}+x_{1}\left(t-\tau_{21}\right)}-a_{22}(t) x_{2}\left(t-\tau_{22}\right)-\frac{a_{23}(t) x_{3}(t)}{m_{2}+x_{2}(t)}\right) \\
& \dot{x}_{3}(t)=x_{3}(t)\left(-a_{3}(t)+\frac{a_{32}(t) x_{2}\left(t-\tau_{32}\right)}{m_{2}+x_{2}\left(t-\tau_{32}\right)}-a_{33}(t) x_{3}\left(t-\tau_{33}\right)\right)
\end{aligned}
$$

In order to consider periodic variations of the environment and the density regulation of the predators though taking into account delay effect and diffusion between patches, more realistic and interesting models of population interactions should take into account comprehensively other than one or two aspects. On the other hand, in order to unify the study of differential and difference equations, people have done a lot of research about dynamic equations on time scales. The principle aim of this paper is to systematically unify the existence of periodic solutions for a delayed ratio-dependent predator-prey system with functional response and diffusion modeled by ordinary differential equations and their discrete analogues in form of difference equations and to extend these results to more general time scales. The approach is based on Gaines and Mawhin's continuation theorem of coincidence degree theory, which has been widely applied to deal with the existence of periodic solutions of differential equations and difference equations.

Therefore, it is interesting and important to study the following model on time scales $\mathbb{T}$ :

$$
\begin{aligned}
z_{1}^{\Delta}(t)= & b_{1}(t)-a_{1}(t) \exp \left\{z_{1}(t)\right\}-\frac{c_{1}(t) \exp \left\{z_{3}(t)\right\}}{m_{1}(t) \exp \left\{z_{3}(t)\right\}+\exp \left\{z_{1}(t)\right\}} \\
& +D_{1}(t)\left(\exp \left\{z_{2}(t)-z_{1}(t)\right\}-1\right), \\
z_{2}^{\Delta}(t)= & b_{2}(t)-a_{2}(t) \exp \left\{z_{2}(t)\right\}+D_{2}(t)\left(\exp \left\{z_{1}(t)-z_{2}(t)\right\}-1\right),
\end{aligned}
$$




$$
\begin{aligned}
z_{3}^{\Delta}(t)= & -r_{1}(t)-a_{3}(t) \exp \left\{z_{3}\left(t-\tau_{11}\right)\right\}+\frac{d_{1}(t) \exp \left\{z_{1}\left(t-\tau_{12}\right)\right\}}{m_{1}(t) \exp \left\{z_{3}\left(t-\tau_{12}\right)\right\}+\exp \left\{z_{1}\left(t-\tau_{12}\right)\right\}} \\
& -\frac{c_{2}(t) \exp \left\{z_{4}(t)\right\}}{m_{2}(t) \exp \left\{z_{4}(t)\right\}+\exp \left\{z_{3}(t)\right\}}, \\
z_{4}^{\Delta}(t)= & -r_{2}(t)-a_{4}(t) \exp \left\{z_{4}\left(t-\tau_{21}\right)\right\}+\frac{d_{2}(t) \exp \left\{z_{3}\left(t-\tau_{22}\right)\right\}}{m_{2}(t) \exp \left\{z_{4}\left(t-\tau_{22}\right)\right\}+\exp \left\{z_{3}\left(t-\tau_{22}\right)\right\}}
\end{aligned}
$$

with the initial conditions

$$
z_{i}(s)=\varphi_{i}(s) \geq 0, \quad s \in[-\tau, 0] \cap \mathbb{T}, \varphi_{i}(0)>0, \varphi_{i}(s) \in C_{\mathrm{rd}}\left([-\tau, 0] \cap \mathbb{T}, \mathbb{R}^{+}\right), i=1,2,3,4,
$$

where $\tau=\max \left\{\tau_{i j}, i, j=1,2\right\}$. In (1.4), $z_{i}(t)$ represents the prey population in the $i$ th patch $(i=1,2)$, and $z_{i}(t)(i=3,4)$ represents the predator population. $z_{1}(t)$ is the prey for $z_{3}(t)$, and $z_{3}(t)$ is the prey for $z_{4}(t)$ so that they form a food chain. $D_{i}(t)$ denotes the dispersal rate of the prey in the $i$ th patch $(i=1,2)$. For the sake of generality and convenience, we always make the following fundamental assumptions for system (1.4):

$(\mathrm{H}) a_{i}(t) \in C_{\mathrm{rd}}\left(\mathbb{T}, \mathbb{R}^{+}\right)(i=1,2,3,4), b_{i}(t), c_{i}(t), d_{i}(t), r_{i}(t), m_{i}(t), D_{i}(t) \in C_{\mathrm{rd}}\left(\mathbb{T}, \mathbb{R}^{+}\right)(i=$ $1,2)$ are all rd-continuous positive periodic functions with period $\omega>0 ; \tau_{i j}(i, j=1,2)$ are nonnegative constants.

In (1.4), set $x_{i}(t)=\exp \left\{z_{i}(t)\right\}, y_{j}(t)=\exp \left\{z_{j+2}(t)\right\}, i=1,2, j=1,2$. If $\mathbb{T}=\mathbb{R}$, then (1.4) reduces to the ratio-dependent predator-prey diffusive system of three species with time delays governed by the ordinary differential equations

$$
\begin{aligned}
& x_{1}^{\prime}(t)=x_{1}(t)\left(b_{1}(t)-a_{1}(t) x_{1}(t)-\frac{c_{1}(t) y_{1}(t)}{m_{1}(t) y_{1}(t)+x_{1}(t)}\right)+D_{1}(t)\left(x_{2}(t)-x_{1}(t)\right), \\
& x_{2}^{\prime}(t)=x_{2}(t)\left(b_{2}(t)-a_{2}(t) x_{2}(t)\right)+D_{2}(t)\left(x_{1}(t)-x_{2}(t)\right), \\
& y_{1}^{\prime}(t)=y_{1}(t)\left(-r_{1}(t)-a_{3}(t) y_{1}\left(t-\tau_{11}\right)+\frac{d_{1}(t) x_{1}\left(t-\tau_{12}\right)}{m_{1}(t) y_{1}\left(t-\tau_{12}\right)+x_{1}\left(t-\tau_{12}\right)}-\frac{c_{2}(t) y_{2}(t)}{m_{2}(t) y_{2}(t)+y_{1}(t)}\right), \\
& y_{2}^{\prime}(t)=y_{2}(t)\left(-r_{2}(t)-a_{4}(t) y_{2}\left(t-\tau_{21}\right)+\frac{d_{2}(t) y_{1}\left(t-\tau_{22}\right)}{m_{2}(t) y_{2}\left(t-\tau_{22}\right)+y_{1}\left(t-\tau_{22}\right)}\right) .
\end{aligned}
$$

If $\mathbb{T}=\mathbb{Z}$, then (1.4) is reformulated as

$$
\begin{aligned}
& x_{1}(k+1)=x_{1}(k) \exp \left\{b_{1}(k)-a_{1}(k) x_{1}(k)-\frac{c_{1}(k) y_{1}(k)}{m_{1}(k) y_{1}(k)+x_{1}(k)}+D_{1}(k)\left(\frac{x_{2}(k)}{x_{1}(k)}-1\right)\right\}, \\
& x_{2}(k+1)=x_{2}(k) \exp \left\{b_{2}(k)-a_{2}(k) x_{2}(k)+D_{2}(k)\left(\frac{x_{1}(k)}{x_{2}(k)}-1\right)\right\},
\end{aligned}
$$




$$
\begin{aligned}
& y_{1}(k+1)=y_{1}(k) \exp \{-r_{1}(k)-a_{3}(k) y_{1}\left(k-\tau_{11}\right)+\frac{d_{1}(k) x_{1}\left(k-\tau_{12}\right)}{m_{1}(k) y_{1}\left(k-\tau_{12}\right)+x_{1}\left(k-\tau_{12}\right)} \\
&\left.-\frac{c_{2}(k) y_{2}(k)}{m_{2}(k) y_{2}\left(k-\tau_{12}\right)+y_{1}\left(k-\tau_{12}\right)}\right\}, \\
& y_{2}(k+1)=y_{2}(k) \exp \left\{-r_{2}(k)-a_{4}(k) y_{2}\left(k-\tau_{21}\right)+\frac{d_{2}(k) y_{1}\left(k-\tau_{22}\right)}{m_{2}(k) y_{2}\left(k-\tau_{22}\right)+y_{1}\left(k-\tau_{22}\right)}\right\}
\end{aligned}
$$

which is the discrete time ratio-dependent predator-prey diffusive system of three species with time delays and is also a discrete analogue of (1.6).

\section{Preliminaries}

A time scale $\mathbb{T}$ is an arbitrary nonempty closed subset of the real numbers $\mathbb{R}$. Throughout the paper, we assume the time scale $\mathbb{T}$ is unbounded above and below, such as $\mathbb{R}, \mathbb{Z}$ and $\cup[2 k, 2 k+1]$. The following definitions and lemmas can be found in [13].

Definition 2.1. The forward jump operator $\sigma: \mathbb{T} \rightarrow \mathbb{T}$, the backward jump operator $\rho: \mathbb{T} \rightarrow$ $\mathbb{T}$, and the graininess $\mu: \mathbb{T} \rightarrow \mathbb{R}^{+}=[0,+\infty)$ are defined, respectively, by

$$
\sigma(t)=\inf \{s \in \mathbb{T} \mid s>t\}, \quad \rho(t)=\sup \{s \in \mathbb{T} \mid s<t\}, \quad \mu(t)=\sigma(t)-t \quad \text { for } t \in \mathbb{T} .
$$

If $\sigma(t)=t$, then $t$ is called right-dense (otherwise: right-scattered), and if $\rho(t)=t$, then $t$ is called left-dense (otherwise: left-scattered).

If $\mathbb{T}$ has a left-scattered maximum $m$, then $\mathbb{T}^{k}=\mathbb{T} \backslash\{m\}$; otherwise $\mathbb{T}^{k}=\mathbb{T}$. If $\mathbb{T}$ has a right-scattered minimum $m$, then $\mathbb{T}_{k}=\mathbb{T} \backslash\{m\}$; otherwise $\mathbb{T}_{k}=\mathbb{T}$.

Definition 2.2. Assume $f: \mathbb{T} \rightarrow \mathbb{R}$ is a function and let $t \in \mathbb{T}^{k}$. Then one defines $f^{\Delta}(t)$ to be the number (provided it exists) with the property that given any $\varepsilon>0$, there is a neighborhood $U$ of $t$ such that

$$
\left|f(\sigma(t))-f(s)-f^{\Delta}(t)(\sigma(t)-s)\right| \leq \varepsilon|\sigma(t)-s| \quad \forall s \in U
$$

In this case, $f^{\Delta}(t)$ is called the delta (or Hilger) derivative of $f$ at $t$. Moreover, $f$ is said to be delta or Hilger differentiable on $\mathbb{T}$ if $f^{\Delta}(t)$ exists for all $t \in \mathbb{T}^{k}$. A function $F: \mathbb{T} \rightarrow \mathbb{R}$ is called an antiderivative of $f: \mathbb{T} \rightarrow \mathbb{R}$ provided $F^{\Delta}(t)=f(t)$ for all $t \in \mathbb{T}^{k}$. Then one defines

$$
\int_{r}^{s} f(t) \Delta t=F(s)-F(r) \quad \text { for } r, s \in \mathbb{T} \text {. }
$$

Definition 2.3. A function $f: \mathbb{T} \rightarrow \mathbb{R}$ is said to be rd-continuous if it is continuous at rightdense points in $\mathbb{T}$ and its left-sided limits exists (finite) at left-dense points in $\mathbb{T}$. The set of rd-continuous functions $f: \mathbb{T} \rightarrow \mathbb{R}$ will be denoted by $C_{\mathrm{rd}}(\mathbb{T}, \mathbb{R})$. 
Definition 2.4. If $a \in \mathbb{T}$, inf $\mathbb{T}=-\infty$, and $f$ is rd-continuous on $(-\infty, a]$, then one defines the improper integral by

$$
\int_{-\infty}^{a} f(t) \Delta t=\lim _{T \rightarrow-\infty} \int_{T}^{a} f(t) \Delta t
$$

provided this limit exists, and one says that the improper integral converges in this case.

Definition 2.5 (see [14]). One says that a time scale $\mathbb{T}$ is periodic if there exists $p>0$ such that if $t \in \mathbb{T}$, then $t \pm p \in \mathbb{T}$. For $\mathbb{T} \neq \mathbb{R}$, the smallest positive $p$ is called the period of the time scale.

Definition 2.6 (see [14]). Let $\mathbb{T} \neq \mathbb{R}$ be a periodic time scale with period $p$. One says that the function $f: \mathbb{T} \rightarrow \mathbb{R}$ is periodic with period $\omega$ if there exists a natural number $n$ such that $\omega=n p, f(t+\omega)=f(t)$ for all $t \in \mathbb{T}$ and $\omega$ is the smallest number such that $f(t+\omega)=f(t)$.

If $\mathbb{T}=\mathbb{R}$, one says that $f$ is periodic with period $\omega>0$ if $\omega$ is the smallest positive number such that $f(t+\omega)=f(t)$ for all $t \in \mathbb{T}$.

Lemma 2.7. Every rd-continuous function has an antiderivative.

Lemma 2.8. Every continuous function is rd-continuous.

Lemma 2.9. If $a, b \in \mathbb{T}, \alpha, \beta \in \mathbb{R}$ and $f, g \in C_{\mathrm{rd}}(\mathbb{T}, \mathbb{R})$, then

(a) $\int_{a}^{b}[\alpha f(t)+\beta g(t)] \Delta t=\alpha \int_{a}^{b} f(t) \Delta t+\beta \int_{a}^{b} g(t) \Delta t$;

(b) if $f(t) \geq 0$ for all $a \leq t<b$, then $\int_{a}^{b} f(t) \Delta t \geq 0$;

(c) if $|f(t)| \leq g(t)$ on $[a, b):=\{t \in \mathbb{T} \mid a \leq t<b\}$, then $\left|\int_{a}^{b} f(t) \Delta t\right| \leq \int_{a}^{b} g(t) \Delta t$.

Lemma 2.10. If $f^{\Delta}(t) \geq 0$, then $f$ is nondecreasing.

Notation 1. To facilitate the discussion below, we now introduce some notation to be used throughout this paper. Let $\mathbb{T}$ be $\omega$-periodic, that is, $t \in \mathbb{T}$ implies $t+\omega \in \mathbb{T}$,

$$
\begin{gathered}
\mathcal{\kappa}=\min \{[0,+\infty) \cap \mathbb{T}\}, \quad I_{\omega}=[\kappa, \kappa+\omega] \cap \mathbb{T}, \\
\bar{f}=\frac{1}{\omega} \int_{I_{\omega}} f(s) \Delta s=\frac{1}{\omega} \int_{\kappa}^{\kappa+\omega} f(s) \Delta s, \quad f^{M}=\sup _{t \in \mathbb{T}} f(t), \quad f^{L}=\inf _{t \in \mathbb{T}} f(t),
\end{gathered}
$$

where $f \in C_{\mathrm{rd}}(\mathbb{T}, \mathbb{R})$ is an $\omega$-periodic function, that is, $f(t+\omega)=f(t)$ for all $t \in \mathbb{T}, t+\omega \in \mathbb{T}$.

Notation 2. Let $X, Z$ be two Banach spaces, let $L:$ Dom $L \subset X \rightarrow Z$ be a linear mapping, and let $N: X \rightarrow Z$ be a continuous mapping. If $L$ is a Fredholm mapping of index zero and there exist continuous projectors $P: X \rightarrow X$ and $Q: Z \rightarrow Z$ such that $\operatorname{Im} P=\operatorname{Ker} L$, $\operatorname{Ker} Q=\operatorname{Im} L=\operatorname{Im}(I-Q)$, then the restriction $\left.L\right|_{\operatorname{Dom} L \cap \operatorname{Ker} P}:(I-P) X \rightarrow \operatorname{Im} L$ is invertible. Denote the inverse of that map by $K_{P}$. If $\Omega$ is an open bounded subset of $X$, the mapping $N$ will be called $L$-compact on $\bar{\Omega}$ if $Q N(\bar{\Omega})$ is bounded and $K_{P}(I-Q) N: \bar{\Omega} \rightarrow \mathrm{X}$ is compact. Since $\operatorname{Im} Q$ is isomorphic to $\operatorname{Ker} L$, there exists an isomorphism $J: \operatorname{Im} Q \rightarrow \operatorname{Ker} L$.

Lemma 2.11 (Continuation theorem [15]). Let X, Z be two Banach spaces, and let $L$ be a Fredholm mapping of index zero. Assume that $N: \bar{\Omega} \rightarrow Z$ is L-compact on $\bar{\Omega}$ with $\Omega$ is open bounded in $X$. 
Furthermore assume the following:

(a) for each $\lambda \in(0,1), x \in \partial \Omega \cap \operatorname{Dom} L, L x \neq \lambda N x$;

(b) for each $x \in \partial \Omega \cap \operatorname{Ker} L, Q N x \neq 0$;

(c) $\operatorname{deg}\{J Q N, \Omega \cap \operatorname{Ker} L, 0\} \neq 0$.

Then the operator equation $L x=N x$ has at least one solution in Dom $L \cap \bar{\Omega}$.

Lemma 2.12 (see [16]). Let $t_{1}, t_{2} \in I_{\omega}$. If $g: \mathbb{T} \rightarrow \mathbb{R}$ is $\omega$-periodic, then

$$
g(t) \leq g\left(t_{1}\right)+\int_{\kappa}^{\kappa+\omega}\left|g^{\Delta}(s)\right| \Delta s, \quad g(t) \geq g\left(t_{2}\right)-\int_{\kappa}^{\kappa+\omega}\left|g^{\Delta}(s)\right| \Delta s
$$

\section{Existence of Periodic Solutions}

The fundamental theorem in this paper is stated as follows about the existence of an $\omega$ periodic solution.

Theorem 3.1. Suppose that $(H)$ holds. Furthermore assume the following:

(i) $b_{i}(t)>D_{i}(t), t \in \mathbb{T}, i=1,2$ ，

(ii) $\bar{b}_{1}-\bar{D}_{1}>\overline{\left(\frac{c_{1}}{m_{1}}\right)}$,

(iii) $\bar{d}_{1}>\bar{r}_{1}+\overline{\left(\frac{c_{2}}{m_{2}}\right)}$,

(iv) $\bar{d}_{2}>\bar{r}_{2}$,

then the system (1.4) has at least one w-periodic solution.

Proof. Consider vector equation

$$
\begin{aligned}
z^{\Delta}(t)= & Y(t), \quad \text { where } z=\left(z_{1}, z_{2}, z_{3}, z_{4}\right)^{T}, z^{\Delta}=\left(z_{1}^{\Delta}, z_{2}^{\Delta}, z_{3}^{\Delta}, z_{4}^{\Delta}\right)^{T}, Y=\left(Y_{1}, Y_{2}, Y_{3}, Y_{4}\right)^{T}, \\
Y_{1}= & b_{1}(t)-D_{1}(t)-a_{1}(t) \exp \left\{z_{1}(t)\right\}-\frac{c_{1}(t) \exp \left\{z_{3}(t)\right\}}{m_{1}(t) \exp \left\{z_{3}(t)\right\}+\exp \left\{z_{1}(t)\right\}} \\
& +D_{1}(t) \exp \left\{z_{2}(t)-z_{1}(t)\right\}, \\
Y_{2}= & b_{2}(t)-D_{2}(t)-a_{2}(t) \exp \left\{z_{2}(t)\right\}+D_{2}(t) \exp \left\{z_{1}(t)-z_{2}(t)\right\}, \\
Y_{3}= & -r_{1}(t)-a_{3}(t) \exp \left\{z_{3}\left(t-\tau_{11}\right)\right\}+\frac{d_{1}(t) \exp \left\{z_{1}\left(t-\tau_{12}\right)\right\}}{m_{1}(t) \exp \left\{z_{3}\left(t-\tau_{12}\right)\right\}+\exp \left\{z_{1}\left(t-\tau_{12}\right)\right\}} \\
& -\frac{c_{2}(t) \exp \left\{z_{4}(t)\right\}}{m_{2}(t) \exp \left\{z_{4}(t)\right\}+\exp \left\{z_{3}(t)\right\}}, \\
Y_{4}= & -r_{2}(t)-a_{4} \exp \left\{z_{4}\left(t-\tau_{21}\right)\right\}+\frac{d_{2}(t) \exp \left\{z_{3}\left(t-\tau_{22}\right)\right\}}{m_{2}(t) \exp \left\{z_{4}\left(t-\tau_{22}\right)\right\}+\exp \left\{z_{3}\left(t-\tau_{22}\right)\right\}} .
\end{aligned}
$$


Define

$$
\begin{gathered}
X=Z=\left\{z \in C_{\mathrm{rd}}\left(\mathbb{T}, \mathbb{R}^{4}\right) \mid z_{i}(t+\omega)=z_{i}(t), i=1,2,3,4, \forall t \in \mathbb{T}\right\}, \\
\|z\|=\left\|\left(z_{1}, z_{2}, z_{3}, z_{4}\right)^{T}\right\|=\sum_{i=1}^{4} \max _{t \in I_{\omega}}\left|z_{i}(t)\right|, \quad z \in X(\text { or } Z),
\end{gathered}
$$

where $|\cdot|$ is the Euclidean norm. Then $X$ and $Z$ are both Banach spaces with the above norm $\|\cdot\|$. Let $N z(t)=Y, L z(t)=z^{\Delta}(t), P z(t)=Q z(t)=\bar{z}, z \in X$. Then

$$
\operatorname{Ker} L=\mathbb{R}^{4}, \quad \operatorname{Im} L=\left\{z \in Z \mid \int_{\kappa}^{\kappa+\omega} z_{i}(t) \Delta t=0, i=1,2,3,4 \text {, for } t \in \mathbb{T}\right\},
$$

and $\operatorname{dim} \operatorname{Ker} L=\operatorname{codim} \operatorname{Im} L=4$. Since $\operatorname{Im} L$ is closed in $X$, then $L$ is a Fredholm mapping of index zero. It is easy to show that $P, Q$ are continuous projectors such that $\operatorname{Im} P=$ $\operatorname{Ker} L, \operatorname{Ker} Q=\operatorname{Im} L=\operatorname{Im}(I-Q)$. Furthermore, the generalized inverse (to $L) K_{P}: \operatorname{Im} L \rightarrow$ Ker $P \cap \operatorname{Dom} L$ exists and is given by $K_{P} z=\int_{\kappa^{t}}^{t} z(s) \Delta s-(1 / \omega) \int_{\mathcal{K}}^{\mathcal{K}+\omega} \int_{\mathcal{K}}^{t} z(s) \Delta s \Delta t$, thus

$$
\begin{gathered}
Q N z=\frac{1}{\omega} \int_{\kappa}^{\kappa+\omega} Y(t) \Delta t \\
K_{P}(I-Q) N z=\int_{\kappa}^{t} Y(s) \Delta s-\frac{1}{\omega} \int_{\kappa}^{\kappa+\omega} \int_{\kappa}^{t} Y(s) \Delta s \Delta t-\left(t-\kappa-\frac{1}{\omega} \int_{\kappa}^{\kappa+\omega}(t-\kappa) \Delta t\right) \bar{Y} .
\end{gathered}
$$

Obviously, $Q N: X \rightarrow Z, K_{P}(I-Q) N: X \rightarrow X$ are continuous. Since $X$ is a Banach space, using the Arzela-Ascoli theorem, it is easy to show that $\overline{K_{P}(I-Q) N(\Omega)}$ is compact for any open bounded set $\Omega \subset X$. Moreover, $Q N(\bar{\Omega})$ is bounded, thus, $N$ is $L$-compact on $\bar{\Omega}$ for any open bounded set $\Omega \subset X$. Corresponding to the operator equation $L z=\lambda N z, \lambda \in(0,1)$, we have

$$
z_{i}^{\Delta}(t)=\lambda Y_{i}(t), \quad i=1,2,3,4
$$

Suppose that $z \in X$ is a solution of (3.5) for certain $\lambda \in(0,1)$. Integrating on both sides of (3.5) from $\kappa$ to $\kappa+\omega$ with respect to $t$, we have

$$
\begin{aligned}
& \int_{\kappa}^{\kappa+\omega}\left(b_{1}(t)-D_{1}(t)\right) \Delta t+\int_{\kappa}^{\kappa+\omega} D_{1}(t) \exp \left\{z_{2}(t)-z_{1}(t)\right\} \Delta t \\
& \quad=\int_{\kappa}^{\kappa+\omega} a_{1}(t) \exp \left\{z_{1}(t)\right\} \Delta t+\int_{\kappa}^{\kappa+\omega} \frac{c_{1}(t) \exp \left\{z_{3}(t)\right\}}{m_{1}(t) \exp \left\{z_{3}(t)\right\}+\exp \left\{z_{1}(t)\right\}} \Delta t \\
& \int_{\kappa}^{\kappa+\omega}\left(b_{2}(t)-D_{2}(t)\right) \Delta t+\int_{\kappa}^{\kappa+\omega} D_{2}(t) \exp \left\{z_{1}(t)-z_{2}(t)\right\} \Delta t \\
& \quad=\int_{\kappa}^{\kappa+\omega} a_{2}(t) \exp \left\{z_{2}(t)\right\} \Delta t
\end{aligned}
$$




$$
\begin{aligned}
\int_{\mathcal{K}}^{\kappa+\omega} & \frac{d_{1}(t) \exp \left\{z_{1}\left(t-\tau_{12}\right)\right\}}{m_{1}(t) \exp \left\{z_{3}\left(t-\tau_{12}\right)\right\}+\exp \left\{z_{1}\left(t-\tau_{12}\right)\right\}} \Delta t \\
= & \bar{r}_{1} \omega+\int_{\mathcal{\kappa}}^{\kappa+\omega} a_{3}(t) \exp \left\{z_{3}\left(t-\tau_{11}\right)\right\} \Delta t \\
& +\int_{\kappa}^{\kappa+\omega} \frac{c_{2}(t) \exp \left\{z_{4}(t)\right\}}{m_{2}(t) \exp \left\{z_{4}(t)\right\}+\exp \left\{z_{3}(t)\right\}} \Delta t \\
\bar{r}_{2} \omega+ & \int_{\mathcal{K}}^{\kappa+\omega} a_{4}(t) \exp \left\{z_{4}\left(t-\tau_{21}\right)\right\} \Delta t \\
= & \int_{\kappa}^{\kappa+\omega} \frac{d_{2}(t) \exp \left\{z_{3}\left(t-\tau_{22}\right)\right\}}{m_{2}(t) \exp \left\{z_{4}\left(t-\tau_{22}\right)\right\}+\exp \left\{z_{3}\left(t-\tau_{22}\right)\right\}} \Delta t .
\end{aligned}
$$

It follows from (3.5) to (3.9) that

$$
\begin{aligned}
& \int_{\kappa}^{\kappa+\omega}\left|z_{1}^{\Delta}(t)\right| \Delta t \leq 2 \int_{\kappa}^{\kappa+\omega} a_{1}(t) \exp \left\{z_{1}(t)\right\} \Delta t+2 \int_{\kappa}^{\kappa+\omega} \frac{c_{1}(t) \exp \left\{z_{3}(t)\right\}}{m_{1}(t) \exp \left\{z_{3}(t)\right\}+\exp \left\{z_{1}(t)\right\}} \Delta t \\
& <2 a_{1}^{M} \int_{\kappa}^{\kappa+\omega} \exp \left\{z_{1}(t)\right\} \Delta t+2 \overline{\left(\frac{c_{1}}{m_{1}}\right)} \omega, \\
& \int_{\kappa}^{\kappa+\omega}\left|z_{2}^{\Delta}(t)\right| \Delta t \leq 2 a_{2}^{M} \int_{\kappa}^{\kappa+\omega} \exp \left\{z_{2}(t)\right\} \Delta t \\
& \int_{\kappa}^{\kappa+\omega}\left|z_{3}^{\Delta}(t)\right| \Delta t \leq 2 \int_{\kappa}^{\kappa+\omega} \frac{d_{1}(t) \exp \left\{z_{1}\left(t-\tau_{12}\right)\right\}}{m_{1}(t) \exp \left\{z_{3}\left(t-\tau_{12}\right)\right\}+\exp \left\{z_{1}\left(t-\tau_{12}\right)\right\}} \Delta t \\
& <2 \bar{d}_{1} \omega=: l_{3}, \\
& \int_{\kappa}^{\kappa+\omega}\left|z_{4}^{\Delta}(t)\right| \Delta t \leq 2 \int_{\kappa}^{\kappa+\omega} \frac{d_{2}(t) \exp \left\{z_{3}\left(t-\tau_{22}\right)\right\}}{m_{2}(t) \exp \left\{z_{4}\left(t-\tau_{22}\right)\right\}+\exp \left\{z_{3}\left(t-\tau_{22}\right)\right\}} \Delta t \\
& <2 \bar{d}_{2} \omega=: l_{4} \text {. }
\end{aligned}
$$

Multiplying (3.6) by $\exp \left\{z_{1}(t)\right\}$ and integrating over $[\kappa, \kappa+\omega]$ gives

$$
\int_{\mathcal{K}}^{\mathcal{\kappa}+\omega} a_{1}(t) \exp \left\{2 z_{1}(t)\right\} \Delta t<\int_{\mathcal{K}}^{\mathcal{\kappa}+\omega}\left(b_{1}(t)-D_{1}(t)\right) \exp \left\{z_{1}(t)\right\} \Delta t+\int_{\mathcal{K}}^{\mathcal{K}+\omega} D_{1}(t) \exp \left\{z_{2}(t)\right\} \Delta t,
$$

which yields

$$
a_{1}^{L} \int_{\kappa}^{\kappa+\omega} \exp \left\{2 z_{1}(t)\right\} \Delta t<\left(b_{1}-D_{1}\right)^{M} \int_{\kappa}^{\kappa+\omega} \exp \left\{z_{1}(t)\right\} \Delta t+D_{1}^{M} \int_{\kappa}^{\kappa+\omega} \exp \left\{z_{2}(t)\right\} \Delta t .
$$


By using the inequality $\left(\int_{\mathcal{K}}^{\kappa+\omega} \exp \left\{z_{1}(t)\right\} \Delta t\right)^{2} \leq \omega \int_{\mathcal{K}}^{\kappa+\omega} \exp \left\{2 z_{1}(t)\right\} \Delta t$, we have

$$
\frac{a_{1}^{L}}{\omega}\left(\int_{\kappa}^{\kappa+\omega} \exp \left\{z_{1}(t)\right\} \Delta t\right)^{2}<\left(b_{1}-D_{1}\right)^{M} \int_{\kappa}^{\kappa+\omega} \exp \left\{z_{1}(t)\right\} \Delta t+D_{1}^{M} \int_{\kappa}^{\kappa+\omega} \exp \left\{z_{2}(t)\right\} \Delta t .
$$

Then

$$
\begin{aligned}
& \frac{2 a_{1}^{L}}{\omega} \int_{\kappa}^{\kappa+\omega} \exp \left\{z_{1}(t)\right\} \Delta t \\
& \quad<\left(b_{1}-D_{1}\right)^{M}+\left\{\left[\left(b_{1}-D_{1}\right)^{M}\right]^{2}+\frac{4 a_{1}^{L} D_{1}^{M}}{\omega} \int_{\kappa}^{\kappa+\omega} \exp \left\{z_{2}(t)\right\} \Delta t\right\}^{1 / 2} .
\end{aligned}
$$

By using the inequality $(a+b)^{1 / 2}<a^{1 / 2}+b^{1 / 2}, a>0, b>0$, we derive from (3.17) that

$$
\frac{a_{1}^{L}}{\omega} \int_{\kappa}^{\kappa+\omega} \exp \left\{z_{1}(t)\right\} \Delta t<\left(b_{1}-D_{1}\right)^{M}+\sqrt{\frac{a_{1}^{L} D_{1}^{M}}{\omega}}\left(\int_{\kappa}^{\kappa+\omega} \exp \left\{z_{2}(t)\right\} \Delta t\right)^{1 / 2} .
$$

Similarly, multiplying (3.7) by $\exp \left\{z_{2}(t)\right\}$ and integrating over $[\kappa, \kappa+\omega]$, then synthesize the above, we obtain

$$
\frac{a_{2}^{L}}{\omega} \int_{\kappa}^{\kappa+\omega} \exp \left\{z_{2}(t)\right\} \Delta t<\left(b_{2}-D_{2}\right)^{M}+\sqrt{\frac{a_{2}^{L} D_{2}^{M}}{\omega}}\left(\int_{\kappa}^{\kappa+\omega} \exp \left\{z_{1}(t)\right\} \Delta t\right)^{1 / 2} .
$$

It follows from (3.18) and (3.19) that

$$
\begin{aligned}
a_{1}^{L} \sqrt{a_{2}^{L}} \int_{\kappa}^{\kappa+\omega} \exp \left\{z_{1}(t)\right\} \Delta t \\
<\omega \sqrt{a_{2}^{L}\left(b_{1}-D_{1}\right)^{M}} \\
\quad+\sqrt{\omega a_{1}^{L} D_{1}^{M}}\left[\sqrt{\omega\left(b_{2}-D_{2}\right)^{M}}+\left(\omega a_{2}^{L} D_{2}^{M} \int_{\kappa}^{\kappa+\omega} \exp \left\{z_{1}(t)\right\} \Delta t\right)^{1 / 4}\right]
\end{aligned}
$$

so, there exists a positive constant $\rho_{1}$ such that

$$
\int_{\kappa}^{\kappa+\omega} \exp \left\{z_{1}(t)\right\} \Delta t<\rho_{1}
$$

which together with (3.19), there also exists a positive constant $\rho_{2}$ such that

$$
\int_{\kappa}^{\kappa+\omega} \exp \left\{z_{2}(t)\right\} \Delta t<\rho_{2}
$$


This, together with (3.11), (3.12), and (3.21), leads to

$$
\begin{gathered}
\int_{\kappa}^{\kappa+\omega}\left|z_{1}^{\Delta}(t)\right| \Delta t<2 a_{1}^{M} \rho_{1}+2 \overline{\left(\frac{c_{1}}{m_{1}}\right)} \omega=: l_{1}, \\
\int_{\kappa}^{\kappa+\omega}\left|z_{2}^{\Delta}(t)\right| \Delta t<2 a_{2}^{M} \rho_{2}=: l_{2} .
\end{gathered}
$$

Since $\left(z_{1}(t), z_{2}(t), z_{3}(t), z_{4}(t)\right)^{T} \in X$, there exist some points $\xi_{i}, \eta_{i} \in I_{\omega}, i=1,2,3,4$, such that

$$
z_{i}\left(\xi_{i}\right)=\min _{t \in I_{\omega}}\left\{z_{i}(t)\right\}, \quad z_{i}\left(\eta_{i}\right)=\max _{t \in I_{\omega}}\left\{z_{i}(t)\right\}, \quad i=1,2,3,4
$$

It follows from (3.21) and (3.22) that

$$
z_{i}\left(\xi_{i}\right)<\ln \frac{\rho_{i}}{\omega}=: L_{i}, \quad i=1,2
$$

From (3.8) and (3.9), we obtain that

$$
z_{3}\left(\xi_{3}\right)<\ln \frac{\bar{d}_{1}-\bar{r}_{1}}{\bar{a}_{3}}=: L_{3}, \quad z_{4}\left(\xi_{4}\right)<\ln \frac{\bar{d}_{2}-\bar{r}_{2}}{\bar{a}_{4}}=: L_{4} .
$$

This, together with (3.12), (3.13), and (3.26), deduces

$$
z_{i}(t) \leq z_{i}\left(\xi_{i}\right)+\int_{\kappa}^{\kappa+\omega}\left|z_{i}^{\Delta}(t)\right| \Delta t<L_{i}+l_{i}, \quad i=1,2,3,4 .
$$

From (3.6) and (3.24), we have

$$
z_{1}\left(\eta_{1}\right) \geq \ln \frac{\bar{b}_{1}-\bar{D}_{1}-\overline{\left(\frac{c_{1}}{m_{1}}\right)}}{\overline{a_{1}}}=: \delta_{1} .
$$

From (3.7) and (3.24), it yields that

$$
z_{2}\left(\eta_{2}\right)>\ln \frac{\bar{b}_{2}-\bar{D}_{2}}{\bar{a}_{2}}=: \delta_{2}
$$


Noticing that $\int_{\mathcal{K}}^{\mathcal{\kappa}+\omega} \exp \left\{z\left(t-\tau_{1}\right)\right\} \Delta t=\int_{\mathcal{K}}^{\mathcal{\kappa}+\omega} \exp \left\{z\left(t-\tau_{2}\right)\right\} \Delta t$, from (3.8) and (3.9), deduces

$$
\begin{aligned}
& \int_{\kappa}^{\kappa+\omega} \frac{d_{1}(t) \exp \left\{z_{1}\left(t-\tau_{12}\right)\right\}}{m_{1}(t) \exp \left\{z_{3}\left(t-\tau_{12}\right)\right\}+\exp \left\{z_{1}\left(t-\tau_{12}\right)\right\}} \Delta t \\
& <\bar{r}_{1} \omega+a_{3}^{M} \int_{\kappa}^{\kappa+\omega} \exp \left\{z_{3}\left(t-\tau_{12}\right)\right\} \Delta t+\overline{\left(\frac{c_{2}}{m_{2}}\right)} \omega, \\
& \int_{\kappa}^{\kappa+\omega} \frac{d_{2}(t) \exp \left\{z_{3}\left(t-\tau_{22}\right)\right\}}{m_{2}(t) \exp \left\{z_{4}\left(t-\tau_{22}\right)\right\}+\exp \left\{z_{3}\left(t-\tau_{22}\right)\right\}} \Delta t \\
& <\bar{r}_{2} \omega+a_{4}^{M} \int_{\kappa}^{\kappa+\omega} \exp \left\{z_{4}\left(t-\tau_{21}\right)\right\} \Delta t .
\end{aligned}
$$

There exist two points $t_{i} \in[\kappa, \kappa+\omega](i=1,2)$ such that

$$
\begin{aligned}
& \frac{d_{1}\left(t_{1}+\tau_{12}\right) \exp \left\{z_{1}\left(t_{1}\right)\right\}}{m_{1}\left(t_{1}+\tau_{12}\right) \exp \left\{z_{3}\left(t_{1}\right)\right\}+\exp \left\{z_{1}\left(t_{1}\right)\right\}}<\bar{r}_{1}+\bar{a}_{3} \exp \left\{z_{3}\left(t_{1}\right)\right\}+\overline{\left(\frac{c_{2}}{m_{2}}\right)}, \\
& \frac{d_{2}\left(t_{2}+\tau_{22}\right) \exp \left\{z_{3}\left(t_{2}\right)\right\}}{m_{2}\left(t_{2}+\tau_{22}\right) \exp \left\{z_{4}\left(t_{2}\right)\right\}+\exp \left\{z_{3}\left(t_{2}\right)\right\}}<\bar{r}_{2} \omega+\bar{a}_{4} \exp \left\{z_{4}\left(t_{2}\right)\right\} .
\end{aligned}
$$

Hence,

$$
\begin{aligned}
& z_{3}\left(t_{1}\right)>\ln \frac{1}{2 \bar{a}_{3} m_{1}^{M}}\{ \sqrt{\left(\bar{r}_{1} m_{1}^{M}+m_{1}^{M} \overline{\left(\frac{c_{2}}{m_{2}}\right)}+\bar{a}_{3} A_{1}\right)^{2}+4 \bar{a}_{3} m_{1}^{M} A_{1}\left(\bar{d}_{1}-\bar{r}_{1}-\overline{\left(\frac{c_{2}}{m_{2}}\right)}\right)} \\
&\left.-\left(\bar{r}_{1} m_{1}^{M}+m_{1}^{M} \overline{\left(\frac{c_{2}}{m_{2}}\right)}+\bar{a}_{3} A_{1}\right)\right\}=: \delta_{3} \\
& z_{4}\left(t_{2}\right)>\ln \frac{\sqrt{\left(\bar{r}_{2} m_{2}^{M}+\bar{a}_{4} A_{2}\right)^{2}+4 \bar{a}_{4} m_{2}^{M} A_{2}\left(\bar{d}_{2}-\bar{r}_{2}\right)}-\left(\bar{r}_{2} m_{2}^{M}+\bar{a}_{4} A_{2}\right)}{2 \bar{a}_{4} m_{2}^{M}}=: \delta_{4}
\end{aligned}
$$

where $A_{1}=\exp \left\{z_{1}\left(\xi_{1}\right)\right\}, A_{2}=\exp \left\{z_{3}\left(\xi_{3}\right)\right\}$. Then, this, together with (3.12), (3.13), (3.23), (3.28), (3.29), and (3.32), deduces

$$
z_{i}(t) \geq z_{i}\left(\eta_{i}\right)-\int_{\kappa}^{\kappa+\omega}\left|z_{i}^{\Delta}(t)\right| \Delta t \geq \delta_{i}-l_{i}, \quad i=1,2,3,4, \text { for any } t \in[\kappa, \kappa+\omega] .
$$

It follows from (3.27) to (3.33) that

$$
\max _{t \in I_{\omega}}\left|z_{i}(t)\right| \leq \max \left\{\left|L_{i}+l_{i}\right|,\left|\delta_{i}-l_{i}\right|\right\}=: B_{i}, \quad i=1,2,3,4
$$


From (3.34), we clearly know that $B_{i}(i=1,2,3,4)$ are independent of $\lambda$, and from the representation of $Q N z$, it is easy to know that there exist points $\zeta_{i} \in[\kappa, \kappa+\omega](i=1,2,3,4)$ such that $Q N z=Y^{*}\left(z_{1}, z_{2}, z_{3}, z_{4}\right)$, where

$$
Y^{*}\left(\begin{array}{c}
z_{1} \\
z_{2} \\
z_{3} \\
z_{4}
\end{array}\right)=\left(\begin{array}{c}
\bar{b}_{1}-\bar{D}_{1}-\bar{a}_{1} \exp \left\{z_{1}\right\}-\frac{\bar{c}_{1} \exp \left\{z_{3}\right\}}{m_{1}\left(\zeta_{1}\right) \exp \left\{z_{3}\right\}+\exp \left\{z_{1}\right\}}-\bar{D}_{1} \exp \left\{z_{2}-z_{1}\right\} \\
\bar{b}_{2}-\bar{D}_{2}-\bar{a}_{2} \exp \left\{z_{2}\right\}+\bar{D}_{2} \exp \left\{z_{1}-z_{2}\right\} \\
-\bar{r}_{1}-\bar{a}_{3} \exp \left\{z_{3}\right\}+\frac{\bar{d}_{1} \exp \left\{z_{1}\right\}}{m_{1}\left(\zeta_{2}\right) \exp \left\{z_{3}\right\}+\exp \left\{z_{1}\right\}}-\frac{\bar{c}_{2} \exp \left\{z_{4}\right\}}{m_{2}\left(\zeta_{3}\right) \exp \left\{z_{4}\right\}+\exp \left\{z_{3}\right\}} \\
-\bar{r}_{2}-\bar{a}_{4} \exp \left\{z_{4}\right\}+\frac{\bar{d}_{2} \exp \left\{z_{3}\right\}}{m_{2}\left(\zeta_{4}\right) \exp \left\{z_{4}\right\}+\exp \left\{z_{3}\right\}}
\end{array}\right) .
$$

Take $B=\sum_{i=0}^{4} B_{i}$, where $B_{0}$ is taken sufficiently large such that $B_{0} \geq \sum_{i=1}^{4}\left|L_{i}\right|+\sum_{i=1}^{4}\left|\delta_{i}\right|$, and such that each solution $u^{*}=\left(u_{1}^{*}, u_{2}^{*}, u_{3}^{*}, u_{4}^{*}\right)^{T}$ of the system $Y^{*}\left(u_{1}, u_{2}, u_{3}, u_{4}\right)^{T}=0$ satisfies $\left\|u^{*}\right\|=\sum_{i=1}^{4}\left|u_{i}^{*}\right|<B_{0}$ if the system (3.35) has solutions. Now take $\Omega=\left\{\left(z_{1}, z_{2}, z_{3}, z_{4}\right)^{T} \in\right.$ $\left.X \mid\left\|\left(z_{1}, z_{2}, z_{3}, z_{4}\right)^{T}\right\|<B\right\}$. Then it is clear that $\Omega$ verifies the requirement (a) of Lemma 2.11.

When $\left(z_{1}, z_{2}, z_{3}, z_{4}\right)^{T} \in \partial \Omega \cap \operatorname{Ker} L=\partial \Omega \cap \mathbb{R}^{4},\left(z_{1}, z_{2}, z_{3}, z_{4}\right)^{T}$ is a constant vector in $\mathbb{R}^{4}$ with $\left\|\left(z_{1}, z_{2}, z_{3}, z_{4}\right)^{T}\right\|=B$, from the definition of $B$, we can naturally derive $Q N z \neq 0$ whether the system (3.35) has solutions or not. This shows that the condition (b) of Lemma 2.11 is satisfied.

Finally, we will prove that the condition (c) of Lemma 2.11 is valid. Define the homotopy $H_{\mu}\left(z_{1}, z_{2}, z_{3}, z_{4}\right): \operatorname{Dom} L \times[0,1] \rightarrow \mathbb{R}^{4}$ by

$$
H_{\mu}\left(z_{1}, z_{2}, z_{3}, z_{4}\right)=\mu Q N\left(z_{1}, z_{2}, z_{3}, z_{4}\right)+(1-\mu) G\left(z_{1}, z_{2}, z_{3}, z_{4}\right), \quad \text { for } \mu \in[0,1]
$$

where

$$
G\left(\begin{array}{c}
z_{1} \\
z_{2} \\
z_{3} \\
z_{4}
\end{array}\right)=\left(\begin{array}{c}
\bar{b}_{1}-\bar{D}_{1}-\bar{a}_{1} \exp \left\{z_{1}\right\} \\
\bar{b}_{2}-\bar{D}_{2}-\bar{a}_{2} \exp \left\{z_{2}\right\} \\
-\bar{r}_{1}-\bar{a}_{3} \exp \left\{z_{3}\right\}+\frac{\bar{d}_{1} \exp \left\{z_{1}\right\}}{m_{1}\left(\zeta_{2}\right) \exp \left\{z_{3}\right\}+\exp \left\{z_{1}\right\}} \\
-\bar{r}_{2}-\bar{a}_{4} \exp \left\{z_{4}\right\}+\frac{\bar{d}_{2} \exp \left\{z_{3}\right\}}{m_{2}\left(\zeta_{4}\right) \exp \left\{z_{4}\right\}+\exp \left\{z_{3}\right\}}
\end{array}\right),
$$

where $\left(z_{1}, z_{2}, z_{3}, z_{4}\right)^{T} \in \mathbb{R}^{4}, \mu \in[0,1]$ is a parameter. From (3.37), it is easy to show that $0 \notin H_{\mu}(\partial \Omega \cap \operatorname{ker} L)$. Moreover, one can easily show that the algebraic equation 


$$
\begin{gathered}
\bar{b}_{1}-\bar{D}_{1}-\bar{a}_{1} u_{1}=0 \\
\bar{b}_{2}-\bar{D}_{2}-\bar{a}_{2} u_{2}=0 \\
-\bar{r}_{1}-\bar{a}_{3} u_{3}+\frac{\bar{d}_{1} u_{1}}{m_{1}\left(\zeta_{2}\right) u_{3}+u_{1}}=0 \\
-\bar{r}_{2}-\bar{a}_{4} u_{4}+\frac{\bar{d}_{2} u_{3}}{m_{2}\left(\zeta_{4}\right) u_{4}+u_{3}}=0
\end{gathered}
$$

has a unique positive solution $\left(u_{1}, u_{2}, u_{3}, u_{4}\right)^{T}$ in $\mathbb{R}_{+}^{4}$. Note that $J=I$ (identical mapping), since $\operatorname{Im} Q=\operatorname{Ker} L$, according to the invariance property of homotopy, direct calculation produces

$$
\begin{aligned}
& \operatorname{deg}\left\{\operatorname{JQN}\left(z_{1}, z_{2}, z_{3}, z_{4}\right)^{T}, \Omega \cap \operatorname{Ker} L,(0,0,0,0)^{T}\right\} \\
& =\operatorname{deg}\left\{G\left(z_{1}, z_{2}, z_{3}, z_{4}\right)^{T}, \Omega \cap \operatorname{Ker} L,(0,0,0,0)^{T}\right\} \\
& \quad=\operatorname{sign}\left|\begin{array}{cccc}
-\bar{a}_{1} u_{1}^{*} & 0 & 0 & 0 \\
0 & -\bar{a}_{2} u_{2}^{*} & 0 & 0 \\
\bar{d}_{1} m_{1}\left(\zeta_{2}\right) u_{3}^{*} & 0 & -\bar{a}_{3}-\frac{\bar{d}_{1} m_{1}\left(\zeta_{2}\right) u_{1}^{*}}{\left(m_{1}\left(\zeta_{2}\right) u_{3}^{*}+u_{1}^{*}\right)^{2}} & 0 \\
\left(m_{1}\left(\zeta_{2}\right) u_{3}^{*}+u_{1}^{*}\right)^{2} & & \frac{\bar{d}_{2} m_{2}\left(\zeta_{4}\right) u_{4}^{*}}{\left(m_{2}\left(\zeta_{4}\right) u_{4}^{*}+u_{3}^{*}\right)^{2}} & -\bar{a}_{4}-\frac{\bar{d}_{2} m_{2}\left(\zeta_{4}\right) u_{3}^{*}}{\left(m_{2}\left(\zeta_{4}\right) u_{4}^{*}+u_{3}^{*}\right)^{2}}
\end{array}\right|=1,
\end{aligned}
$$

where $\operatorname{deg}\{\cdot, \cdot, \cdot\}$ is the Brouwer degree. By now we have proved that $\Omega$ verifies all requirements of Lemma 2.11. Therefore, (1.4) has at least one $\omega$-periodic solution in Dom $L \cap$ $\bar{\Omega}$. The proof is complete.

Corollary 3.2. If the conditions in Theorem 3.1 hold, then both the corresponding continuous model (1.6) and the discrete model (1.7) have at least one w-periodic solution.

Remark 3.3. If $\mathbb{T}=\mathbb{R}$ and $\tau_{11} \equiv \tau_{21} \equiv 0$ in (1.6), then the system (1.6) reduces to the continuous ratio-dependence predator-prey diffusive system proposed in [17].

Remark 3.4. If we only consider the prey population in one-patch environment and ignore the dispersal process in the system (1.4), then the classical ratio-dependence two species predator-prey model in particular of (1.4) with Michaelis-Menten functional response and time delay on time scales

$$
\begin{aligned}
& z_{1}^{\Delta}(t)=r_{1}(t)-a(t) \exp \left\{z_{1}(t)\right\}-\frac{c(t) \exp \left\{z_{2}(t)\right\}}{m(t) \exp \left\{z_{2}(t)\right\}+\exp \left\{z_{1}(t)\right\}} \\
& z_{2}^{\Delta}(t)=-r_{2}(t)+\frac{d(t) \exp \left\{z_{1}(t-\tau)\right\}}{m(t) \exp \left\{z_{2}(t-\tau)\right\}+\exp \left\{z_{1}(t-\tau)\right\}}
\end{aligned}
$$


where $a(t), c(t), d(t), r_{i}(t), m(t) \in C_{\mathrm{rd}}\left(\mathbb{T}, \mathbb{R}^{+}\right)(i=1,2)$ are positive $\omega$-periodic functions, $\tau$ is nonnegative constant. It is easy to obtain the corresponding conclusions on time scales for the system (3.40).

Corollary 3.5. Suppose that (i) $\bar{r}_{1}>\overline{\left(\frac{c}{m}\right)}$, (ii) $d(t)>r_{2}(t), t \in \mathbb{T}$ hold, then (3.40) has at least one w-periodic solution.

Remark 3.6. The result in Corollary 3.5 is same as those for the corresponding continuous and discrete systems.

\section{Acknowledgments}

The author is very grateful to his supervisor Prof. M. Fan and the anonymous referees for their many valuable comments and suggestions which greatly improved the presentation of this paper. This work is supported by the Foundation for subjects development of Harbin University (no. HXK200716) and by the Foundation for Scientific Research Projects of Education Department of Hei-longjiang Province of China (no. 11513043).

\section{References}

[1] E. Beretta and Y. Kuang, "Convergence results in a well-known delayed predator-prey system," Journal of Mathematical Analysis and Applications, vol. 204, no. 3, pp. 840-853, 1996.

[2] K. Gopalsamy, Stability and Oscillations in Delay Differential Equations of Population Dynamics, vol. 74 of Mathematics and Its Applications, Kluwer Academic Publishers, Dordrecht, The Netherlands, 1992.

[3] Y. Kuang, Delay Differential Equations with Applications in Population Dynamics, vol. 191 of Mathematics in Science and Engineering, Academic Press, Boston, Mass, USA, 1993.

[4] Y. Kuang, "Rich dynamics of Gause-type ratio-dependent predator-prey system," in Differential Equations with Applications to Biology (Halifax, NS, 1997), vol. 21 of Fields Institute Communications, pp. 325-337, American Mathematical Society, Providence, RI, USA, 1999.

[5] R. Arditi and L. R. Ginzburg, "Coupling in predator-prey dynamics: ratio-dependence," Journal of Theoretical Biology, vol. 139, no. 3, pp. 311-326, 1989.

[6] M. Fan and K. Wang, "Periodicity in a delayed ratio-dependent predator-prey system," Journal of Mathematical Analysis and Applications, vol. 262, no. 1, pp. 179-190, 2001.

[7] M. Fan and Q. Wang, "Periodic solutions of a class of nonautonomous discrete time semi-ratiodependent predator-prey systems," Discrete and Continuous Dynamical Systems. Series B, vol. 4, no. 3, pp. 563-574, 2004.

[8] S.-B. Hsu, T.-W. Hwang, and Y. Kuang, "Global analysis of the Michaelis-Menten-type ratiodependent predator-prey system," Journal of Mathematical Biology, vol. 42, no. 6, pp. 489-506, 2001.

[9] C. Jost, O. Arino, and R. Arditi, "About deterministic extinction in ratio-dependent predator-prey models," Bulletin of Mathematical Biology, vol. 61, no. 1, pp. 19-32, 1999.

[10] D. Xiao and S. Ruan, "Multiple bifurcations in a delayed predator-prey system with nonmonotonic functional response," Journal of Differential Equations, vol. 176, no. 2, pp. 494-510, 2001.

[11] R. Xu and L. Chen, "Persistence and global stability for a three-species ratio-dependent predator-prey system with time delays in two-patch environments," Acta Mathematica Scientia. Series B, vol. 22, no. 4, pp. 533-541, 2002.

[12] H.-F. Huo and W.-T. Li, "Periodic solution of a delayed predator-prey system with Michaelis-Menten type functional response," Journal of Computational and Applied Mathematics, vol. 166, no. 2, pp. 453 463, 2004.

[13] M. Bohner and A. Peterson, Dynamic Equations on Time Scales: An Introduction with Applications, Birkhäuser, Boston, Mass, USA, 2001.

[14] E. R. Kaufmann and Y. N. Raffoul, "Periodic solutions for a neutral nonlinear dynamical equation on a time scale," Journal of Mathematical Analysis and Applications, vol. 319, no. 1, pp. 315-325, 2006.

[15] R. E. Gaines and J. L. Mawhin, Coincidence Degree, and Nonlinear Differential Equations, vol. 568 of Lecture Notes in Mathematics, Springer, Berlin, Germany, 1977. 
[16] M. Bohner, M. Fan, and J. Zhang, "Existence of periodic solutions in predator-prey and competition dynamic systems," Nonlinear Analysis: Real World Applications, vol. 7, no. 5, pp. 1193-1204, 2006.

[17] S. Wen, S. Chen, and H. Mei, "Positive periodic solution of a more realistic three-species LotkaVolterra model with delay and density regulation," Chaos, Solitons and Fractals. In press. 